TITLE:

Reducing delays to prothrombin complex concentrate administration in vitamin $\mathrm{K}$ antagonist-related intracerebral haemorrhage: a quality improvement initiative

\title{
AUTHOR DETAILS:
}

Elizabeth Marrinan, Core Medical Trainee ${ }^{1}$

Lucia Chen, Core Medical Trainee ${ }^{1}$

David Werring, Professor, Neurology Consultant ${ }^{2}$

David Turner, Stroke and Geriatric Consultant ${ }^{2}$

${ }^{1}$ University College London Hospital, London

${ }^{2}$ National Hospital for Neurology and Neurosurgery, London

\section{ABSTRACT}

Four-factor prothrombin complex concentrate (PCC) is the first-line treatment in Vitamin-K antagonist related intracerebral haemorrhage (VKA-ICH). Early administration is associated with improved patient outcomes.

We investigated delays in PCC administration in VKA-ICH in order to reduce the time from CT scan confirming ICH to PCC administration (scan-to-needle time).

20 patients were identified by retrospective audit over a three-year period. The median scan-to-needle time for PCC was 156 minutes. Several points of delay were identified, including contacting both Haematology and Transfusion departments for PCC dosing and dispensing. 
Following this audit, our interventions included the introduction of a protocol with a PCC dosing algorithm, negating the need to contact Haematology prior to administration. A dedicated supply of PCC was given to the Stroke Unit avoiding the need to contact the transfusion service.

Re-audit showed a 68\% reduction in median scan-to-needle time from 156 minutes to 49 minutes. Prospective data collection is ongoing.

\section{INTRODUCTION}

Vitamin-K antagonist (VKA) related intracerebral haemorrhage (ICH) accounts for around $10-20 \%$ of all ICH (Parry-Jones et al. 2015) and carries a worse prognosis than ICH unrelated to VKA due to a doubled risk of haematoma expansion (Brouwers et al. 2014). Haematoma expansion in ICH is an important indicator of neurological deterioration and a poor prognostic factor (Davis et al. 2006). VKA-ICH has a high case fatality. In patients with INR between 2 and 3 at the time of $\mathrm{ICH}$, 3-month mortality rate is 52\% (Meretoja et al. 2012). Four-factor prothrombin complex concentrate (PCC) is currently recommended as first-line treatment in VKAICH (National clinical guideline for stroke, 2016) as it is the most effective agent to achieve timely reversal of VKA and reduction of International Normalized Ratio (INR) (Steiner et al. 2016). Speed of PCC administration is important because the faster the reduction in INR, the lower the risk of haematoma expansion (Kuramatsu et 
al. 2015). The need for rapid treatment of acute ischaemic stroke is now well established, with protocols for administration of key therapies including intravenous thrombolysis and thrombectomy. However, there is less recognition of the potential importance of rapid treatment in patients with $\mathrm{ICH}$.

Reducing door-to-needle PCC administration times has become a focus for improving outcomes for ICH patients. A recent quality improvement initiative achieved a 50\% reduction in door-to-needle times for PCC administration and significant reduction in 30-day case fatality in $\mathrm{ICH}$ patients by implementing three main changes within the stroke service: point-of-care INR testing, immediate supply of PCC in the emergency department, and a VKA-ICH protocol that negated the need for discussion with Haematologists (Parry-Jones 2015; Paroutoglou and Parry-Jones 2018).

Many of these implemented changes are potentially translatable to other centres. We therefore developed our own Quality Improvement initiative to improve the management of VKA-ICH by reducing PCC door-toneedle times at the University College London Hospital (UCLH) Hyper-Acute Stroke Unit. We built on the existing strategy by also implementing simulation training for VKA-ICH treatment.

THE AUDIT

\section{Methods}

We identified 30 patients between October 2012 and October 2015 who had radiological evidence of intracerebral haemorrhage on computed tomography (CT) who were taking VKA and who had received sameday PCC treatment. Clinical notes, radiology and laboratory results were reviewed and data collected on: demographics, time of admission, time of CT head, point-of-care INR result, laboratory INR result, Vitamin $\mathrm{K}$ administration time, PCC administration time, and details of any relevant delays.

\section{Results}


Full clinical records were available for 20 patients. The mean age at presentation was 77 years with 11 (55\%) male and 9 (45\%) female patients. A point of care INR was documented in 9 cases (45\%) with a mean value of 2.8. Laboratory INR on admission was available in 16 cases (80\%) with a mean value of 3.0. Admission INR was not available for two patients because the sample haemolysed, for one patient the sample was under-filled and one patient did not have an INR sent on admission. Point-of-care INR was documented in nine cases with a mean value of 2.8 (1dp) (range $2-4)$.

The median time from arrival in A\&E to CT scan was 32 minutes (range 11 to 222 minutes). The median time from CT scan confirming haemorrhage to vitamin K administration was 64 minutes (range 18 to 420 minutes); the median time from scan confirming haemorrhage to prothrombin complex concentrate administration was 156 minutes (range 63 to 540 minutes). In three cases prothrombin complex concentrate was given after transfer to the surgical intensive care unit at the National Hospital for Neurology and Neurosurgery (NHNN). Their transfer to a critical care unit was considered higher priority than reversal. Nine patients died as a result of intracerebral haemorrhage.

\section{THE INTERVENTION}

Our primary aim was to reduce delays in the reversal of VKA in patients presenting with an intracerebral haemorrhage. Based on the results of the audit we identified three key interventions.

Firstly, we introduced a new protocol for managing anticoagulant-associated intracerebral haemorrhage. Importantly this protocol included a clear prothrombin complex concentrate dosing algorithm. It is also stated in the protocol that VKA reversal should take place prior to any transfer to neurosurgical intensive care at NHNN. (Figure 1)

Secondly, we sought to eliminate delays in administration of prothrombin complex concentrate and vitamin K. After discussion with the Haematology department we organised a dedicated Hyper-acute Stroke Unit supply 
of prothrombin complex concentrate, rather than needing to contact the transfusion service to dispense the drug. Vitamin $\mathrm{K}$ was also added to the thrombolysis bag which is brought to each thrombolysis call.

Thirdly, simulation training on management of VKA-ICH was introduced to fully embed this protocol amongst staff and to increase awareness of the urgency of VKA reversal in $\mathrm{ICH}$. This training was given to doctors and nurses in the Hyper-acute Stroke Unit. There was further training for thrombolysis nurses including demonstration vials of PCC to familiarise nurses with drug preparation.

\section{THE RE-AUDIT}

\section{Methods}

For the re-audit, data collection was prospective over an 11-month period (from July 2016 to May 2017) on the Hyper-acute Stroke Unit at UCLH. All patients who received PCC were included in the study.

As previously, the following information was obtained from patient notes and the electronic results system: demographics, time of admission, time of CT head, point-of-care INR result, laboratory INR result, Vitamin $\mathrm{K}$ administration time, PCC administration time, and details of any relevant delays.

\section{Results}

Following the introduction of our interventions, we conducted prospective data collection over an 11-month period (July 2016 to May 2017). Twelve patients presented with VKA-ICH during this period. The median scan to needle time for PCC fell from 156 minutes (pre-intervention) to 49 minutes (a 68\% reduction). In cases where transfer to a neurosurgical centre was required, all patients received PCC at UCLH prior to transfer to NHNN. The median scan to vitamin $\mathrm{K}$ administration time was 53 minutes post intervention (64 minutes pre-intervention). (Figure 2) 


\section{DISCUSSION}

The results of our initial audit were striking in that no patients received PCC in under 60 minutes following identification of $\mathrm{ICH}$. We believe that the main factors contributing to this delay were: limited knowledge regarding the urgency of VKA reversal in VKA-ICH despite rapid diagnosis by $\mathrm{CT}$ scan; the requirement for case-by-case discussions with the Haematology department for advice on PCC dosing and for authorisation of PCC use; and a lack of familiarity amongst nursing staff in preparing and administering PCC due to the infrequency of VKA-ICH (around once per month). Furthermore, in three cases, delays were due to the need for transfer to a critical care unit, which was considered higher priority than VKA-reversal.

With the aim of reducing delays in administration of PCC in cases of $\mathrm{ICH}$, we introduced several interventions. In line with those introduced in the successful Salford Royal quality improvement initiative (Parry-Jones 2015) we introduced a VKA-ICH protocol that negated the need for discussion with haematologists and organised an immediate supply of PCC within the hyper-acute stroke unit. This removed the need for PCC to be couriered from the transfusion lab which is off site. In addition to the above we increased awareness of the importance of rapid VKA reversal in patients with VKA-ICH and introduced a VKA-ICH scenario into simulation training attended by both current and new members of staff. VKA-ICH simulation training has been repeated several times throughout the year and has formed part of the induction for new staff joining the department.

We have shown that improvement in time to PCC administration in cases of VKA-ICH can be achieved in a central London Hyper-acute Stroke Unit. The VKA-ICH protocol has undergone subsequent review and has been adapted to include guidance on managing ICH in patients receiving direct oral anticoagulants (DOACs). The pragmatic steps of securing a dedicated supply of PCC and a HASU-specific protocol can reduce scan-toneedle times, and we anticipate that this will be applicable to cases of ICH in patients taking DOACs. As the 
number of patients with atrial fibrillation on anticoagulation rises, improving the management of ICH in this setting is essential (Lane et al. 2017).

Prospective data collection is ongoing to monitor whether the positive changes we have seen in the period studied are sustained in the longer term. We hope that by continued education of staff through regular simulation sessions we can ensure that staff are equipped to manage these cases efficiently and safely.

\section{KEYWORDS}

Intracerebral haemorrhage; Prothrombin complex concentrate; Stroke; Vitamin K antagonist; Warfarin

\section{KEY POINTS}

1. VKA-ICH is associated with a high case fatality rate. Reducing the rate of haematoma expansion by prompt administration of PCC is associated with improved outcomes. 
2. We identified multiple factors contributing to delays in the administration of PCC in VKA-ICH at a tertiary UK Hyper-Acute Stroke Unit.

3. Introduction of a protocol including guidance on dosing of PCC negated the need to consult Haematology prior to administering PCC, while providing a dedicated Stroke Unit supply of PCC avoided the need to consult the transfusion laboratory in the acute setting. Furthermore, staff attended VKA-ICH simulation training to fully embed the protocol in the department.

4. Through a combination of the above interventions, we achieved a $68 \%$ median reduction in scan-toneedle time from 156 to 49 minutes for PCC administration in VKA-ICH. Ongoing prospective data collection is in place to ensure that this positive change is sustained.

\section{REFERENCES}

Brouwers HB, Chang Y, Falcone GJ, et al. Predicting hematoma expansion after primary intracerebral hemorrhage. JAMA Neurology. 2014 Feb. 71(2): 158-64.

Davis SM, Broderick J, Hennerici M et al. Hematoma growth is a determinant of mortality and poor outcome after intracerebral hemorrhage. Neurology. 2006 Apr 25. 66(8): 1175 - 81. 
Kuramatsu JB, Gerner ST, Schellinger PD et al. Anticoagulant reversal, blood pressure levels, and anticoagulant resumption in patients with anticoagulation-related intracerebral hemorrhage. JAMA. 2015 Feb 24. 313(8):

$824-36$.

Lane DA, Skjoth F, Lip GYH et al. Temporal trends in incidence, prevalence, and mortality of atrial fibrillation in primary care. J Am Heart Assoc. 2017 Apr 28;6(5). Pii: e005155.

Meretoja A, Strbian D, Putaala J, et al. SMASH-U: A proposal for etiologic classification of intracerebral hemorrhage. Stroke. 2012 Oct. 43(10):2592-7.

National clinical guideline for stroke, 5th edition. Intercollegiate Stroke Working Party. London: Royal College of Physicians. 2016.

Paroutoglou K, Parry-Jones A. Hyperacute management of intracerebral haemorrhage. Clin Med (Lond). 2018 Apr. 18(2): s9-s12.

Parry-Jones A. Cutting delays in reversing anticoagulation after intracerebral haemorrhage: three key changes at a UK comprehensive stroke centre. BMJ Qual Improv Rep. 2015 Jun. 4(1): u208763.w3521.

Parry-Jones AR, Di Napoli M, Goldstein JN et al. Reversal strategies for vitamin K antagonists in acute intracerebral hemorrhage. Ann Neurol. 2015 Jul. 78(1): 54-62.

Steiner T, Poli S, Griebe M et al. Fresh frozen plasma versus prothrombin complex concentrate in patients with intracranial haemorrhage related to vitamin $\mathrm{K}$ antagonists (INCH): a randomised trial. Lancet Neurol. 2016 May. 15(6): $566-73$. 
